\title{
Anticancer therapy: boosting the bang of Bim
}

\author{
Andrea Wahner Hendrickson, Xue Wei Meng, and Scott H. Kaufmann \\ Department of Oncology and Department of Molecular Pharmacology, Mayo Clinic, Rochester, Minnesota, USA.
}

\begin{abstract}
Even though activating mutations of B-Raf, a kinase atop the MAPK signaling cascade, reportedly sensitize tumor cells to MEK inhibitors, Raf and MEK inhibitors have exhibited limited clinical activity. In this issue of the JCI, Cragg et al. report that MEK inhibition upregulates the proapoptotic Bcl-2 family member Bim but induces little regression of human melanoma xenografts in mice unless the Bcl-2 antagonist ABT-737 is added (see the related article, doi:10.1172/JCI35437). These findings illustrate the potential benefit of simultaneously inhibiting oncogenic kinases and inhibiting Bcl-2 action in solid tumors.
\end{abstract}

\section{Oncogenic activation of the Raf/MEK/ERK pathway}

Over the past decade, an increasingly detailed understanding of the molecular pathogenesis of cancer has led to identification of a variety of new targets for anticancer drugs. Two of the best-studied signaling pathways that are activated by oncogenic kinase mutations are the MAPK pathway and the PI3K/Akt pathway (Figure 1). According to current understanding, the former pathway involves signaling from a variety of receptor tyrosine kinases through adaptor molecules that activate the small GTPase Ras. Upon activation, Ras binds and activates members of the Raf serine/threonine protein kinase family, thereby triggering sequential phosphorylation and activation of the MEK kinases and their downstream targets ERK1 and ERK2. Upon activation, ERK1 and ERK2 phosphorylate a number of cytoplasmic and nuclear substrates involved in cell survival and proliferation. In the nucleus, ERK-mediated phosphorylation of c-myc, ELK1, and other transcription factors leads to increased expression of genes involved in cell cycle progression (1). In the cytoplasm, ERK-induced phosphorylation of Bcl-2 family members has been reported to inhibit apoptosis, as described below.

In addition to the MAPK pathway, receptor tyrosine kinases and Ras activate PI3K, which generates the lipid second messenger phosphatidylinositol-3,4,5-trisphospate

Nonstandard abbreviations used: $\mathrm{BH} 3, \mathrm{Bcl}-2$ homology 3.

Conflict of interest: The authors have declared that no conflict of interest exists.

Citation for this article: J. Clin. Invest. doi:10.1172/ JCI37553.
(PIP3), again setting into motion a protein kinase signaling cascade. PIP3 activates the serine/threonine kinase phosphoinositidedependent kinase 1 , which catalyzes the turn phosphorylates a number of proteins (e.g., the cyclin-dependent kinase inhibitor $\mathrm{p} 27^{\mathrm{Kip} 1}$ ) that regulate cell cycle progression as well as transcription factors (e.g., NF- $\kappa \mathrm{B}$, Foxo3a) and other molecules that limit susceptibility of cells to apoptosis.

Studies performed over the past decade have revealed many ways in which one or both of these pathways are activated in tumors. Signaling is initiated not only by mutations that lock Ras in its GTP-bound (i.e., activated) state, but also by mutations of receptor tyrosine kinases such as EGFR and the EGFR family member HER2/Neu. Particularly pertinent to the present discussion are the more recently described activating mutations of $B-R A F$, which occur in almost $70 \%$ of melanomas, $30 \%$ of thyroid cancers, $15 \%$ of ovarian cancers, and $10 \%$ of colorectal cancers (2, 3 ). Whereas activating mutations of EGFR confer hypersensitivity to EGFR tyrosine kinase inhibitors such as gefitinib (4), $B-R A F$ mutations have been reported to uniquely confer sensitivity to MEK inhibitors (5). Despite these observations, clinical studies of MEK and Raf inhibitors have yielded relatively disappointing results, even in patients with mutations that activate the MAPK pathway $(3,6,7)$. While it is clearly possible for MEK inhibitors to inhibit growth of xenografts with activating $B$-RAF mutations (5), tumor regressions have been the exception rather than the rule in preclinical models and in the clinical setting, raising concern that some other pathway also needs to be modulated in order to facilitate tumor shrinkage. activating phosphorylation of Akt. Akt in

\section{Effects of MAPK pathway activation on Bcl-2 family members}

In addition to enhancing cell proliferation, the MAPK pathway also regulates the mitochondrial pathway of apoptosis, a pathway in which the oncoprotein $\mathrm{Bcl}-2$ and related proteins play a prominent role (8-10). Based on structural and functional criteria, members of this protein family can be subdivided into 3 classes. The first class, which contains $\mathrm{Bcl}-2, \mathrm{Bcl}-\mathrm{x}_{\mathrm{L}}, \mathrm{Mcl}-1, \mathrm{Bcl}-\mathrm{w}$, and A1, inhibits apoptosis by binding to proapoptotic $\mathrm{Bcl}-2$ family members. The second class includes Bax and Bak, which are involved in releasing proapoptotic proteins from mitochondria, possibly by forming pores in the outer mitochondrial membrane. The third class, called Bcl-2 homology 3-only (BH3-only) proteins, includes Bim, Bad, Puma, Noxa, Bmf, and several other family members, all of which contain a 9- to 15 -amino acid $\mathrm{BH} 3$ domain that is thought to be important in binding and neutralizing antiapoptotic Bcl-2 family members.

The BH3-only proteins appear to serve as molecular stress sensors within cells (9). Two of the family members, Noxa and Puma, are transcriptionally upregulated in response to DNA damage and other stimuli. Other family members such as Bim are constitutively expressed but sequestered by binding to polypeptides in various cellular compartments. In response to various stresses (e.g., cytoskeletal disruption or loss of growth signals), specific $\mathrm{BH} 3$-only proteins are released and activated. At least 2 models have been proposed to explain the subsequent induction of apoptosis (8-10). One model focuses on the purported ability of some of these polypeptides to directly activate Bax and Bak, thereby causing release of cytochrome $c$ from mitochondria (10). The other focuses exclusively on the ability of all of these family members to bind and inactivate antiapoptotic Bcl-2 molecules (8).

The activities of Bcl-2 family members are regulated, in part, by posttranslational modifications. Antiapoptotic kinases, for example, catalyze activating phosphorylations of Bcl-2 $(11,12)$ and $\operatorname{Mcl}-1(13,14)$ as well as inactivating phosphorylations of $\operatorname{Bad}$ and $\operatorname{Bim}(15,16)$. While some of these phosphorylations are mediated through 


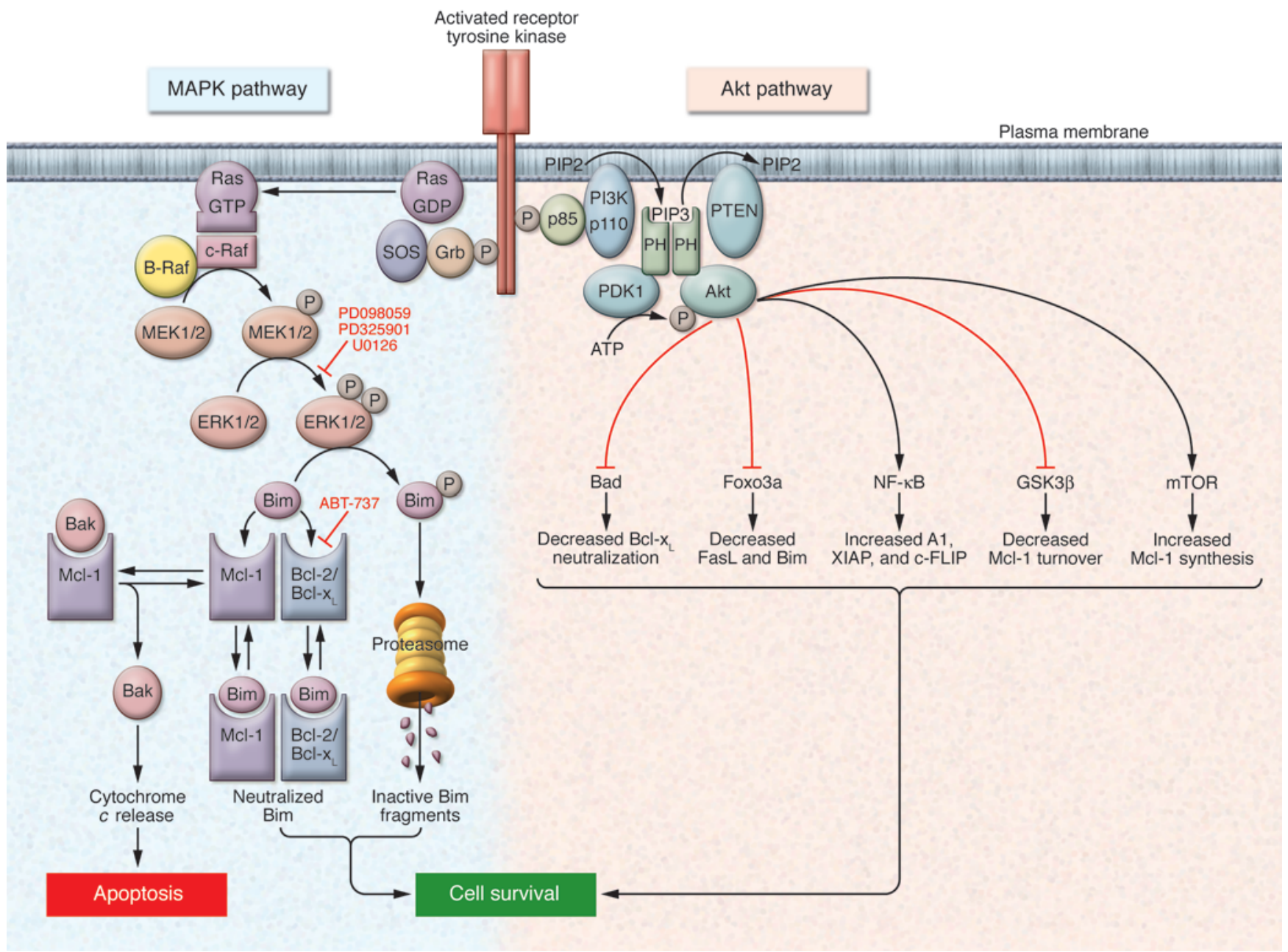

Figure 1

Overview of signaling pathways affected by MEK inhibitor/ABT-737 combination therapy. In healthy cells, mitogenic growth factors signaling through the MAPK pathway trigger sequential phosphorylation and activation of MEK and ERK kinases, which in turn leads to stabilization of Mcl-1, activation of $\mathrm{Bcl}-2$, and degradation of Bim, thereby promoting cell survival. In tumors with B-RAF mutations, B-Raf causes pathway activation independent of upstream signaling by either directly activating MEK1/2 or activating c-Raf, depending on the B-Raf variant present. In this issue of the $\mathrm{JCl}$, Cragg et al. show that MEK inhibitors such as U0126, PD098059, and PD325901 upregulate Bim but do not induce regressions of tumors harboring $B-R A F$ mutations (17). The authors also propose that $\mathrm{Bcl}-2$ and $\mathrm{Bcl}-\mathrm{x}_{\mathrm{L}}$ neutralize the increased Bim, thereby diminishing the potential apoptotic effect of the MEK inhibitors. Consistent with this model, the authors show that addition of the Bcl-2/Bcl- $x_{L}$ antagonist ABT-737 enhances the apoptotic effect of MEK inhibition. When Bcl-2 and Bcl- $\mathrm{x}_{\mathrm{L}}$ are antagonized, the upregulated Bim binds to $\mathrm{Mcl}-1$, causing it to release Bak and/or Bax, leading to cytochrome $c$ release and subsequent apoptosis. Because tumors with $B-R A F$ mutations are dependent on the MAPK pathway rather than the Akt pathway for survival, combining a MEK inhibitor and a $\mathrm{Bcl}-2 / \mathrm{Bcl}-\mathrm{x}_{\mathrm{L}}$ antagonist appears to be a promising strategy for treating these tumors. c-FLIP, cellular FLICE (caspase 8) inhibitory protein; Grb, growth factor receptor-bound protein 2; GSK, glycogen synthase kinase; mTOR, mammalian target of rapamycin; PDK1, phosphoinositide-dependent kinase 1; PH, pleckstrin homology domain; PIP2, phosphoinositol-4,5-bisphosphate; PIP3, phosphoinositol-3,4,5-trisphosphate; PTEN, phosphatase and tensin homolog; SOS, son of sevenless; XIAP, X-linked inhibitor of apoptosis protein.

the Akt pathway, others clearly involve ERK1/2 or their immediate downstream target $\mathrm{p} 90$ ribosomal $\mathrm{S} 6$ kinase.

\section{Enhancing the effects of upregulated Bim}

Building on previous reports showing that ERK-mediated Bim phosphorylation leads to proteasome-mediated Bim degradation $(15,16)$, Cragg et al. report in the current issue of the JCI that MEK inhibition leads to Bim upregulation in a variety of $B-R A F$ mutant human melanoma and colon cancer cell lines (17). Interestingly, however, the MEK inhibitors induce modest apoptosis in vitro and exhibit little antitumor effect on melanoma xenografts in mice in vivo. Results of further experiments suggest that the upregulated Bim is bound and presumably neutralized by $\mathrm{Bcl}-2$ and $\mathrm{Bcl}-\mathrm{x}_{\mathrm{L}}$
(Figure 1). Consistent with this interpretation, Cragg et al. show that the $\mathrm{BH} 3$ mimetic ABT-737, which binds to and inhibits Bcl-2 and $\mathrm{BCl}-\mathrm{x}_{\mathrm{L}}$, increases MEK inhibitor-induced apoptosis in vitro and slows tumor growth - sometimes dramatically - in vivo.

\section{Questions and future perspectives}

The present study by Cragg et al. (17) represents an elegant step forward in translating 
current understanding of apoptotic pathways into potentially improved therapies. Nonetheless, important questions remain. The mechanistic basis for the apparent synergy of MEK inhibitors and a $\mathrm{BH} 3$ mimetic remains incompletely understood. While the results reported by Cragg et al. show that Bim shRNA protects cells from MEK inhibitor-induced death at early time points (17), Bim shRNA is much less protective with extended treatment. These observations raise the possibility that a second cytotoxic mechanism might be triggered during prolonged MEK inhibition. Earlier observations that the Raf/MEK/ERK pathway regulates stability of the antiapoptotic protein $\mathrm{Mcl}-1(14,18)$, as well as antiapoptotic activity of Bcl-2 itself $(11,12)$, raise the possibility that Mcl-1 downregulation and/or Bcl-2 dephosphorylation might also contribute to the cytotoxic effects of MEK inhibition. A possible contribution from interruption of ERK-mediated phosphorylation of other Bcl-2 family members also remains to be investigated. Finally, the effects of these agents on normal cells require further study. Although it is convenient to invoke the idea that selectivity comes from the phenomenon of oncogene addiction (19), i.e., the dependence of a cancer cell on one overactive gene or pathway for its survival and growth, the apoptosis resulting from drug-induced Bim upregulation combined with Bcl-2 inhibition described by Cragg et al. might be predicted to cause substantial toxicity in normal cells as well.

Compared with certain other attempts to alter Bcl-2 family members for therapeutic benefit (20), the effects of combined MEK inhibitor/BH3 mimetic therapy appear particularly promising. However, the ability to translate these findings into the clinic will depend on the toxicities encountered. The dose-limiting toxicities of MEK inhibition appear to be rash and hypoxia (1), but the dose-limiting toxicities of $\mathrm{BH} 3$ mimetics in humans have not yet been reported. Although the toxicities of this therapeutic combination in mice were reportedly tolerable in the current study (17), it remains to be seen whether these classes of agents can be combined at therapeutic doses and without toxic side effects in the clinic.
In view of the potential benefit of simultaneously inhibiting the MAPK pathway and antagonizing $\mathrm{Bcl}-2$ in tumors with $B-R A F$ mutations, it is reasonable to ask whether this approach will also work in tumors driven by other oncogenic kinases. Additional studies from the same research group indicate that the $\mathrm{BCR} / \mathrm{ABL}$ kinase inhibitor imatinib and the EGFR tyrosine kinase inhibitor gefitinib also induce apoptosis, at least in part, through Bim upregulation (ref. 17 and references therein). Because $\mathrm{BCR} / \mathrm{ABL}$ and EGFR activate signaling through the Akt pathway as well as the MAPK pathway (Figure 1), it is slightly surprising that Bim upregulation is the only prominent change in Bcl-2 family members observed with MEK, BCR/ABL, and EGFR inhibitors. In all cases, however, ABT-737 enhances the effect of the kinase inhibitor. Accordingly, one wonders whether the MEK inhibitor/BH3 mimetic therapy would also be active in other tumors that exhibit MAPK activation in the absence of $B-R A F$ mutations.

Finally, while there is some evidence that MEK might represent a better target than Raf (3), the Raf inhibitor sorafenib is in fact approved for certain therapeutic indications, raising the question of what would happen if tumors harboring $B-R A F$ mutations were treated with ABT-737 plus sorafenib rather than experimental MEK inhibitors. Answers to all of these questions are awaited with interest.

\section{Acknowledgments}

We thank members of the Kaufmann laboratory for stimulating discussions and Deb Strauss for editorial assistance. Work in the authors' laboratory is supported in part by NIH grant R01 CA69008.

Address correspondence to: Scott H. Kaufmann, Division of Oncology Research, Guggenheim 1342C, Mayo Clinic, 200 First St. SW, Rochester, Minnesota 55905, USA. Phone: (507) 284-8950; Fax: (507) 284-3906; E-mail: kaufmann.scott@mayo.edu.

Andrea Wahner Hendrickson and Xue Wei Meng contributed equally to this work.
1. Wang, J.Y., Wilcoxen, K.M., Nomoto, K., and Wu, S. 2007. Recent advances of MEK inhibitors and their clinical progress. Curr. Top. Med. Chem. 7:1364-1378.

2. Tuveson, D.A., Weber, B.L., and Herlyn, M. 2003. $\mathrm{BRAF}$ as a potential therapeutic target in melanoma and other malignancies. Cancer Cell. 4:95-98.

3. Madhunapantula, S.V., and Robertson, G.P. 2008. Is B-Raf a good therapeutic target for melanoma and other malignancies? Cancer Res. 68:5-8.

4. Dancey, J.E. 2004. Predictive factors for epidermal growth factor receptor inhibitors - the bull's-eye hits the arrow. Cancer Cell. 5:411-415.

5. Solit, D.B., et al. 2006. BRAF mutation predicts sensitivity to MEK inhibition. Nature. 439:358-362.

6. Rinehart, J., et al. 2004. Multicenter phase II study of the oral MEK inhibitor, CI-1040, in patients with advanced non-small-cell lung, breast, colon, and pancreatic cancer. J. Clin. Oncol. 22:4456-4462.

7. Eisen, T., et al. 2006. Sorafenib in advanced melanoma: a Phase II randomised discontinuation trial analysis. Br. J. Cancer. 95:581-586.

8. Adams, J.M., and Cory, S. 2007. Bcl-2-regulated apoptosis: mechanism and therapeutic potential. Curr. Opin. Immunol. 19:488-496.

9. Youle, R.J., and Strasser, A. 2008. The BCL-2 protein family: opposing activities that mediate cell death. Nat. Rev. Mol. Cell Biol. 9:47-59.

10. Chipuk, J.E., and Green, D.R. 2008. How do BCL-2 proteins induce mitochondrial outer membrane permeabilization? Trends Cell Biol. 18:157-164.

11. Ito, T., Deng, X., Carr, B., and May, W.S. 1997. Bcl-2 phosphorylation required for anti-apoptosis function. J. Biol. Chem. 272:11671-11673.

12. Deng, X., Gao, F., Flagg, T., and May, W.S., Jr. 2004. Mono- and multisite phosphorylation enhances Bcl2's antiapoptotic function and inhibition of cell cycle entry functions. Proc. Natl. Acad. Sci. U. S. A. 101:153-158.

13. Kobayashi, S., et al. 2007. Serine 64 phosphorylation enhances the antiapoptotic function of Mcl-1. J. Biol. Chem. 282:18407-18417.

14. Domina, A.M., Vrana, J.A., Gregory, M.A., Hann, S.R., and Craig, R.W. 2004. MCL1 is phosphorylated in the PEST region and stabilized upon ERK activation in viable cells, and at additional sites with cytotoxic okadaic acid or taxol. Oncogene. 23:5301-5315.

15. Ley, R., et al. 2004. Extracellular signal-regulated kinases $1 / 2$ are serum-stimulated Bim $_{\mathrm{EL}}$ causing its phosphorylation and turnover. J. Biol. Chem. 279:8837-8847.

16. Luciano, F., et al. 2003. Phosphorylation of Bim-El by Erk1/2 on serine 69 promotes its degradation via the proteasome pathway and regulates its proapoptotic function. Oncogene. 22:6785-6793.

17. Cragg, M.S., et al. 2008. Treatment of B-RAF mutant human tumor cells with a MEK inhibitor requires Bim and is enhanced by a $\mathrm{BH} 3$ mimetic. J. Clin. Invest. 118:3651-3659.

18. Meng, X., et al. 2003. Central role of FADD in apoptosis induction by the mitogen-activated protein kinase kinase inhibitor CI1040 (PD184352) in acute lymphocytic leukemia cell lines in vitro. J. Biol. Chem. 278:47326-47339.

19. Weinstein, I.B., and Joe, A. 2008. Oncogene addiction. Cancer Res. 68:3077-3080; discussion 3080.

20. Walensky, L.D., et al. 2004. Activation of apoptosis in vivo by a hydrocarbon-stapled BH3 helix. Science. 305:1466-1470. 